\title{
The Genomic Formation of South and Central Asia
}

Authors: Vagheesh M. Narasimhan ${ }^{1, *}$, Nick Patterson ${ }^{2,3, *}$, Priya Moorjani ${ }^{4,5,+}$, Iosif Lazaridis ${ }^{1}$, Mark Lipson ${ }^{1}$, Swapan Mallick ${ }^{1,2,6}$, Nadin Rohland ${ }^{1,2}$, Rebecca Bernardos ${ }^{1}$, Alexander M. $\mathrm{Kim}^{1,7}$, Nathan Nakatsuka ${ }^{1,8}$, Iñigo Olalde ${ }^{1}$, Alfredo Coppa ${ }^{9}$, James Mallory ${ }^{10}$, Vyacheslav Moiseyev ${ }^{11}$, Janet Monge ${ }^{12}$, Luca M. Olivieri ${ }^{13}$, Nicole Adamski ${ }^{1,6}$, Nasreen Broomandkhoshbacht ${ }^{1,6}$, Francesca Candilio ${ }^{12,14,15}$, Olivia Cheronet ${ }^{14,16,17}$, Brendan J. Culleton $^{18,19}$, Matthew Ferry ${ }^{1,6}$, Daniel Fernandes ${ }^{14,16,17,20}$, Beatriz Gamarra ${ }^{14,16}$, Daniel Gaudio ${ }^{14}$, Mateja Hajdinjak ${ }^{21}$, Éadaoin Harney ${ }^{1,6,22}$, Thomas K. Harper ${ }^{18,19}$, Denise Keating ${ }^{14}$, Ann Marie Lawson ${ }^{1,6}$, Megan Michel ${ }^{1,6,23}$, Mario Novak ${ }^{14,24}$, Jonas Oppenheimer ${ }^{1,6}$, Niraj Rai ${ }^{25,26}$, Kendra Sirak $^{14,27}$, Viviane Slon ${ }^{21}$, Kristin Stewardson ${ }^{1,6}$, Zhao Zhang ${ }^{1}$, Gaziz Akhatov ${ }^{28}$, Anatoly N. Bagashev $^{29}$, Bauryzhan Baitanayev ${ }^{28}$, Gian Luca Bonora ${ }^{30}$, Tatiana Chikisheva ${ }^{31}$, Anatoly Derevianko $^{31}$, Enshin Dmitry ${ }^{29}$, Katerina Douka ${ }^{32,33}$, Nadezhda Dubova ${ }^{34}$, Andrey Epimakhov $^{35,36}$, Suzanne Freilich ${ }^{17}$, Dorian Fuller ${ }^{37}$, Alexander Goryachev ${ }^{29}$, Andrey Gromov ${ }^{11}$, Bryan Hanks ${ }^{38}$, Margaret Judd ${ }^{38}$, Erlan Kazizov ${ }^{28}$, Aleksander Khokhlov ${ }^{39}$, Egor Kitov ${ }^{34}$, Elena Kupriyanova $^{41}$, Pavel Kuznetsov ${ }^{39}$, Donata Luiselli ${ }^{42}$, Farhod Maksudov ${ }^{43}$, Christopher Meiklejohn ${ }^{44}$, Deborah Merrett ${ }^{45}$, Roberto Micheli ${ }^{13,46}$, Oleg Mochalov ${ }^{39}$, Zahir Muhammed ${ }^{32,47}$, Samariddin Mustafokulov ${ }^{43,48}$, Ayushi Nayak ${ }^{32}$, Rykun M. Petrovna ${ }^{49}$, Davide Pettener ${ }^{42}$, Richard Potts ${ }^{50}$, Dmitry Razhev ${ }^{29}$, Stefania Sarno ${ }^{42}$, Kulyan Sikhymbaeva ${ }^{40}$, Sergey M. Slepchenko ${ }^{29}$, Nadezhda Stepanova ${ }^{31}$, Svetlana Svyatko ${ }^{10,51}$, Sergey Vasilyev ${ }^{34}$, Massimo Vidale $^{13,52}$, Dmitriy Voyakin ${ }^{28,53}$, Antonina Yermolayeva ${ }^{28}$, Alisa Zubova ${ }^{11,31}$, Vasant S. Shinde ${ }^{54}$, Carles Lalueza-Fox ${ }^{55}$, Matthias Meyer ${ }^{21}$, David Anthony ${ }^{56}$, Nicole Boivin ${ }^{32,+}$, Kumarasamy Thangaraj ${ }^{25,+}$, Douglas J. Kennett ${ }^{18,19,+}$, Michael Frachetti ${ }^{57,58,+}$, Ron Pinhasi ${ }^{14,17,+}$, David Reich $1,2,6,59,+$

* Contributed equally

+ Co-directed this work

To whom correspondence should be addressed: V.N. (vagheesh@mail.harvard.edu), N.P. (nickp@broadinstitute.org), or D.R. (reich@genetics.med.harvard.edu)

\section{Affiliations}

${ }^{1}$ Department of Genetics, Harvard Medical School, Boston, MA 02115, USA

${ }^{2}$ Broad Institute of Harvard and MIT, Cambridge, MA 02142, USA

${ }^{3}$ Radcliffe Institute for Advanced Study, Harvard University, Cambridge, MA 02138, USA

${ }^{4}$ Department of Molecular and Cell Biology, University of California, Berkeley, CA 94720, USA

${ }^{5}$ Center for Computational Biology, University of California, Berkeley, CA 94720, USA

${ }^{6}$ Howard Hughes Medical Institute, Harvard Medical School, Boston, MA 02115, USA

${ }^{7}$ Department of Anthropology, Harvard University, Cambridge, MA 02138, USA

${ }^{8}$ Harvard-MIT Division of Health Sciences and Technology, Harvard Medical School, Boston, MA 02115, USA

${ }^{9}$ Dipartimento di Biologia Ambientale, Sapienza Università di Roma, Rome 00185, Italy

${ }^{10}$ School of Natural and Built Environment, Queen's University Belfast, Belfast BT7 1NN, Northern Ireland, UK 
${ }^{11}$ Peter the Great Museum of Anthropology and Ethnography (Kunstkamera), Russian Academy of Science, St. Petersburg 199034, Russia

${ }^{12}$ University of Pennsylvania Museum of Archaeology and Anthropology, Philadelphia, PA 19104, USA

${ }^{13}$ ISMEO Italian Archaeological Mission in Pakistan, 19200 Saidu Sharif (Swat), Pakistan

${ }^{14}$ Earth Institute, University College Dublin, Dublin 4, Ireland

${ }^{15}$ Soprintendenza Archeologia, Belle Arti e Paesaggio per la Città Metropolitana di Cagliari e le Province di Oristano e Sud Sardegna, Cagliari 09124, Italy

${ }^{16}$ School of Archaeology, University College Dublin, Dublin 4, Ireland

${ }^{17}$ Department of Anthropology, University of Vienna, 1090 Vienna, Austria

${ }^{18}$ Department of Anthropology, Pennsylvania State University, University Park, PA 16802, USA

${ }^{19}$ Institutes for Energy and the Environment, Pennsylvania State University, University Park, PA 16802, USA

${ }^{20}$ CIAS, Department of Life Sciences, University of Coimbra, Coimbra 3000-456, Portugal

${ }^{21}$ Max Planck Institute for Evolutionary Anthropology, Leipzig 04103, Germany

${ }^{22}$ Department of Organismic and Evolutionary Biology, Harvard University, Cambridge, MA 02138, USA

${ }^{23}$ Department of Human Evolutionary Biology, Harvard University, Cambridge MA, 02138, USA

${ }^{24}$ Institute for Anthropological Research, Zagreb 10000, Croatia

${ }^{25}$ CSIR-Centre for Cellular and Molecular Biology, Hyderabad 500 007, India

${ }^{26}$ Birbal Sahni Institute of Palaeosciences, Lucknow 226007, India

${ }^{27}$ Department of Anthropology, Emory University, Atlanta, GA 30322, USA

${ }^{28}$ Institute of Archaeology A.Kh. Margulan, Almaty 050010, Kazakhstan

${ }^{29}$ Tyumen Scientific Centre SB RAS, Institute of the Problems of Northern Development, Tyumen 625003, Russia

${ }^{30}$ Archaeology of Asia Department, ISMEO - International Association of Mediterranean and Oriental Studies, Rome RM00186, Italy

${ }^{31}$ Institute of Archaeology and Ethnography, Siberian Branch, Russian Academy of Sciences, Novosibirsk 630090, Russia

${ }^{32}$ Department of Archaeology, Max Planck Institute for the Science of Human History, Jena 07745, Germany

${ }^{33}$ Oxford Radiocarbon Accelerator Unit, Research Laboratory for Archaeology and the History of Art, University of Oxford, Oxford OX1 3QY, UK

${ }^{34}$ Institute of Ethnology and Anthropology, Russian Academy of Sciences, Moscow 119991, Russia

${ }^{35}$ Institute of History and Archaeology, Ural Branch RAS, Yekaterinburg 620990, Russia

${ }^{36}$ South Ural State University, Chelyabinsk 454080, Russia

${ }^{37}$ Institute of Archaeology, University College London, London WC1H OPY, UK

${ }^{38}$ University of Pittsburgh, Department of Anthropology, Pittsburgh, PA 15260, USA

${ }^{39}$ Samara State University of Social Sciences and Education, Samara 443099, Russia

${ }^{40}$ Central State Museum Republic of Kazakhstan, Samal-1 Microdistrict, Almaty 050010, Kazakhstan

${ }^{41}$ Scientific and Educational Center of Study on the Problem of Nature and Man, Chelyabinsk State University, Chelyabinsk 454021, Russia 
$91{ }^{42}$ Department of Biological, Geological and Environmental Sciences, Alma Mater Studiorum -

92 University of Bologna, Bologna 40126, Italy

$93{ }^{43}$ Institute for Archaeological Research, Uzbekistan Academy of Sciences, Samarkand 140151,

94 Uzbekistan

$95{ }^{44}$ Department of Anthropology, University of Winnipeg, Winnipeg, MB, R3B 2E9, Canada

$96{ }^{45}$ Department of Archaeology, Simon Fraser University, Burnaby, BC, V5A 1S6, Canada

$97{ }^{46}$ MiBACT - Soprintendenza Archeologia, Belle Arti e Paesaggio del Friuli Venezia Giulia,

$98 \quad 34135$ Trieste, Italy

$99 \quad{ }^{47}$ Department of Archaeology, Hazara University, Mansehra 21300, Pakistan

$100 \quad{ }^{48}$ Afrosiab Museum, Samarkand 140151, Uzbekistan

$101{ }^{49}$ Tomsk State National Research University, Tomsk 634050, Russia

$102{ }^{50}$ Human Origins Program, National Museum of Natural History, Smithsonian Institution,

103 Washington, DC 20013, USA

$104{ }^{51}$ CHRONO Centre for Climate, the Environment, and Chronology, Queen's University of

105 Belfast, Belfast BT7 1NN, Northern Ireland, UK

$106 \quad{ }^{52}$ Department of Cultural Heritage: Archaeology and History of Art, Cinema and Music,

107 University of Padua, Padua 35139, Italy

$108{ }^{53}$ Archaeological Expertise LLP, Almaty 050060, Kazakhstan

$109{ }^{54}$ Department of Archaeology, Deccan College Post-Graduate and Research Institute, Pune

$110 \quad 411006$, India

$111{ }^{55}$ Institute of Evolutionary Biology, CSIC-Universitat Pompeu Fabra, Barcelona 08003, Spain

$112 \quad{ }^{56}$ Anthropology Department, Hartwick College, Oneonta, New York 13820, USA

$113{ }^{57}$ Department of Anthropology, Washington University in St. Louis, St. Louis, MO 63112, USA

$114{ }^{58}$ Spatial Analysis, Interpretation, and Exploration Laboratory, Washington University in St.

115 Louis, St. Louis, MO 63112, USA

$116{ }^{59}$ Max Planck-Harvard Research Center for the Archaeoscience of the Ancient Mediterranean,

117 Cambridge, MA 02138, USA 


\section{Abstract}

119 The genetic formation of Central and South Asian populations has been unclear because of an 120 absence of ancient DNA. To address this gap, we generated genome-wide data from 362 ancient

121 individuals, including the first from eastern Iran, Turan (Uzbekistan, Turkmenistan, and

122 Tajikistan), Bronze Age Kazakhstan, and South Asia. Our data reveal a complex set of genetic

123 sources that ultimately combined to form the ancestry of South Asians today. We document a

124 southward spread of genetic ancestry from the Eurasian Steppe, correlating with the

125 archaeologically known expansion of pastoralist sites from the Steppe to Turan in the Middle

126 Bronze Age (2300-1500 BCE). These Steppe communities mixed genetically with peoples of the

127 Bactria Margiana Archaeological Complex (BMAC) whom they encountered in Turan (primarily

128 descendants of earlier agriculturalists of Iran), but there is no evidence that the main

129 BMAC population contributed genetically to later South Asians. Instead, Steppe communities

130 integrated farther south throughout the 2nd millennium BCE, and we show that they mixed with

131 a more southern population that we document at multiple sites as outlier individuals exhibiting a

132 distinctive mixture of ancestry related to Iranian agriculturalists and South Asian hunter-gathers.

133 We call this group Indus Periphery because they were found at sites in cultural contact with the

134 Indus Valley Civilization (IVC) and along its northern fringe, and also because they were

135 genetically similar to post-IVC groups in the Swat Valley of Pakistan. By co-analyzing ancient

136 DNA and genomic data from diverse present-day South Asians, we show that Indus Periphery-

137 related people are the single most important source of ancestry in South Asia — consistent with

138 the idea that the Indus Periphery individuals are providing us with the first direct look at the

139 ancestry of peoples of the IVC - and we develop a model for the formation of present-day South

140 Asians in terms of the temporally and geographically proximate sources of Indus Periphery-

141 related, Steppe, and local South Asian hunter-gatherer-related ancestry. Our results show how

142 ancestry from the Steppe genetically linked Europe and South Asia in the Bronze Age, and

143 identifies the populations that almost certainly were responsible for spreading Indo-European

144 languages across much of Eurasia.

146 One Sentence Summary: Genome wide ancient DNA from 357 individuals from Central and

147 South Asia sheds new light on the spread of Indo-European languages and parallels between the 148 genetic history of two sub-continents, Europe and South Asia. 
Main text

\section{Ancient DNA Data and Analysis Strategy}

We generated whole-genome ancient DNA data from 362 previously unreported ancient individuals and higher quality data from 17 previously reported individuals. Almost all derive from three broad regions: 132 from Iran and the southern part of Central Asia (present-day Turkmenistan, Uzbekistan, and Tajikistan, which we call Turan; "Iran/Turan"), 165 from the western and central Steppe and northern forest zone encompassing present day Kazakhstan and Russia ("Forest Zone/Steppe"), and 65 from northern Pakistan ("South Asia"). Our dataset includes the first published ancient DNA data from 1) Chalcolithic and Bronze Age eastern Iran and Turan (5600-1200 BCE from 12 sites); 2) early ceramic-using hunter-gatherers from the western Siberian forest zone (6200-4000 BCE from 2 sites); 3) Chalcolithic and Bronze Age pastoralists from the Steppe east of the Ural mountains, including the first ancient data from

163 Bronze Age Kazakhstan (4700-1000 BCE from 20 sites); and 4) the first ever ancient DNA from

164 South Asia from Iron Age and historical settlements in the Swat Valley of Pakistan (1200 BCE -

1651 CE from 7 sites) (Fig. 1, Supplementary Materials, Data S1). To generate these data, we

166 prepared samples in dedicated clean rooms, extracted DNA $(1,2)$, constructed libraries for

167 Illumina sequencing $(3,4)$, and screened them using previously described procedures $(5-7)$. We

168 enriched the libraries for DNA overlapping around 1.24 million single nucleotide polymorphisms

169 (SNPs), sequenced the products on Illumina instruments, and performed quality control (Data

170 S1) $(5,6,8)$. We also report 186 new direct radiocarbon dates on human bone (Data S2). After

171 grouping individuals based on archaeological and chronological information and merging with

172 previously reported data, our dataset included 612 ancient individuals that we then co-analyzed

173 with genome-wide data from present-day individuals genotyped at around 600,000 SNPs, 1,789

174 of which were from 246 ethnographically-distinct groups in South Asia (Data S3;

175 Supplementary Materials) (9-11). We restricted analyses to ancient samples covered by at least

17615,000 SNPs. We use Italic font to refer to genetic groupings and normal font to indicate

177 archaeological cultures or sites.

179 We carried out principal component analysis (PCA) by projecting the ancient individuals onto 180 the patterns of genetic variation in present-day Eurasians (Fig. 1) $(12,13)$. This revealed three 
181 major groupings, closely corresponding to the geographic regions of the Forest Zone/Steppe,

182 Iran/Turan and South Asia, a pattern we replicate in ADMIXTURE clustering (14). To test

183 formally whether populations differ significantly in their ancestry within regions, we used

184 symmetry- $f_{4}$-statistics measuring whether pairs of populations differ in their degree of allele

185 sharing to a third population, and admixture- $f_{3}$-statistics to test formally for mixture

186 (Supplementary Materials). We tested the fit of mixture models using qpAdm, which evaluates

187 whether all possible $f_{4}$-statistics relating a set of tested populations to outgroup populations is

188 consistent with mixtures of a pre-specified number of sources and if so estimates proportions of

189 ancestry (5). We can model almost every population as a mixture of seven deeply divergent

190 "distal" ancestry sources (usually closely related to populations for which we have data, but in

191 some cases deeply related):

192

193 - "Anatolian agriculturalist-related": represented by $7^{\text {th }}$ millennium BCE western Anatolian

194 agriculturalists (6)

- "Western European Hunter-Gatherer (WHG)-related": represented by Mesolithic western

196 Europeans $(5,10,15,16)$

197 - "Iranian agriculturalist-related": represented by $8^{\text {th }}$ millennium BCE pastoralists from the

198 Zagros Mountains of Iran $(17,18)$

199 • "Eastern European Hunter-Gatherer ( $E H G)$-related": represented by hunter-gatherers from

200 diverse sites in Eastern Europe $(5,6)$

201 • "West Siberian Hunter-Gatherer (West_Siberian_HG)-related": a newly documented deep

202 source of Eurasian ancestry represented here by three samples

203 • "East Asian-related": represented in this study by Han Chinese

- "Ancient Ancestral South Indian ( $A A S I)$-related": a hypothesized South Asian Hunter-Gatherer

205 lineage related deeply to present-day indigenous Andaman Islanders (19)

207 We also used $q p A d m$ to identify "proximal" models for each group as mixtures of temporally

208 preceding groups. This often identified multiple alternative models that were equally good fits to

209 the data. These analyses were nevertheless useful because we could identify patterns that were

210 qualitatively consistent across models. The discussion that follows presents an overview of these 
211 analyses, while the Supplementary Materials presents the full details. Table 1 summarizes the

212 key findings that emerge from our analysis.

\section{Iran/Turan}

215 We analyzed our newly generated data together with previously published data to examine the

216 genetic transformations that accompanied the spread of agriculture eastward from Iran beginning

217 in the $7^{\text {th }}$ millennium $\operatorname{BCE}(20,21)$. Our analysis confirms that early Iranian agriculturalists from

218 the Zagros Mountains harbor a distinctive type of West Eurasian ancestry $(17,18)($ Fig. 1), while

219 later groups across a broad geographic region were admixed between this type of ancestry and

220 that related to early Anatolian agriculturalists. (In this paper we use the term "agriculturalists" to

221 refer both to crop cultivation and/or herding, and accordingly refer to the people of the Zagros

222 Mountains who kept domesticated goats as agriculturalists $(17,22,23)$.) We show that there was

223 a west-to-east cline of decreasing Anatolian agriculturalist-related admixture ranging from $\sim 70 \%$

224 in Chalcolithic Anatolia to $\sim 33 \%$ in eastern Iran, to $\sim 3 \%$ in far eastern Turan (Fig. 1;

225 Supplementary Materials). The timing of the establishment of this cline is consistent with the

226 dates of spread of wheat and barley agriculture from west to east (in the $7^{\text {th }}$ to $6^{\text {th }}$ millennia

227 BCE), suggesting the possibility that individuals of Anatolian ancestry may have contributed to

228 spreading agriculturalist economies not only westward to Europe, but also eastward to Iran (21,

229 24, 25). An increase of Anatolian agriculturalist-related ancestry was also proposed for the Pre-

230 Pottery agriculturalists from the Levant in comparison to the earlier Natufian hunter-gatherers

231 (17), further supporting this hypothesis. However, without data on the distribution of

232 Anatolian/Iranian-agriculturalist ancestry in early agriculturalists in Mesopotamia, it is difficult

233 to determine when the cline was established. In the far eastern part of this cline (eastern Iran and

234 Turan) we also detect admixture related to West_Siberian_HG, proving that North Eurasian

235 admixture impacted Turan well before the spread of Yamnaya-related Steppe pastoralists

236 (Steppe_EMBA).

238 From Bronze Age Turan, we report 69 ancient individuals (2300-1400 BCE) from four urban

239 sites of the Bactria Margiana Archaeological Complex (BMAC) and its immediate successors.

240 The great majority of individuals fall in a genetic cluster that is similar, albeit not identical, to the 241 preceding groups in Turan in harboring a large proportion of early Iranian agriculturalist-related 
ancestry ( $\sim 60 \%$ in the $B M A C)$ with smaller components of Anatolian agriculturalist-related

243 ancestry $(\sim 21 \%)$ and West_Siberian_HG-related ancestry $(\sim 13 \%)$ suggesting that the main

$244 B M A C$ cluster coalesced from preceding pre-urban populations in Turan (which in turn likely

245 derived from earlier eastward spreads from Iran). The absence in the $B M A C$ cluster of the

246 Steppe_EMBA ancestry that is ubiquitous in South Asia today_along with qpAdm analyses that

247 rule out $B M A C$ as a substantial source of ancestry in South Asia (Fig. 3A) — suggests that while

248 the BMAC was affected by the same demographic forces that later impacted South Asia (the

249 southward movement of Middle to Late Bronze Age Steppe pastoralists described in the next

250 section), it was also bypassed by members of these groups who hardly mixed with $B M A C$ people

251 and instead mixed with peoples further south. In fact, the data suggest that instead of the main

$252 B M A C$ population having a demographic impact on South Asia, there was a larger effect of gene

253 flow in the reverse direction, as the main $B M A C$ genetic cluster is slightly different from the

254 preceding Turan populations in harboring 5\% of their ancestry from the AASI.

256 We also observe outlier individuals at multiple sites, revealing interactions among populations

257 that would be difficult to appreciate without the large sample sizes reported here.

259 First, around 2300 BCE in Turan, we observe two outliers at the BMAC site of Gonur with

260 West_Siberian_HG-related ancestry of a type that we observe at multiple sites in Kazakhstan

261 over the preceding and succeeding millennia. The most plausible explanation is that this ancestry

262 is that of indigenous populations associated with the Kelteminar culture, the native hunter-

263 gatherers of the region who covered a vast area of Central Asia before the BMAC (26). Future

264 ancient DNA data from Kelteminar contexts will make it possible to determine whether it is

265 indeed the case that the genetic ancestry of Kelteminar people was similar to that of

266 West_Siberia_HG. Importantly, in the $3^{\text {rd }}$ millenium BCE we do not find any individuals with

267 ancestry derived from Yamnaya-related Steppe pastoralists in Turan. Thus, Steppe_EMBA

268 ancestry was not yet widespread across the region.

270 Second, between 2100-1700 BCE, we observe BMAC outliers from three sites with

271 Steppe_EMBA ancestry in the admixed form typically carried by the later Middle to Late Bronze

272 Age Steppe groups (Steppe_MLBA). This documents a southward movement of Steppe ancestry 
273 through this region that only began to have a major impact around the turn of the $2^{\text {nd }}$ millennium

274 BCE.

275

276 Third, between 3100-2200 BCE we observe an outlier at the BMAC site of Gonur, as well as two

277 outliers from the eastern Iranian site of Shahr-i-Sokhta, all with an ancestry profile similar to 41

278 ancient individuals from northern Pakistan who lived approximately a millennium later in the

279 isolated Swat region of the northern Indus Valley (1200-800 BCE). These individuals had

280 between $14-42 \%$ of their ancestry related to the AASI and the rest related to early Iranian

281 agriculturalists and West_Siberian_HG. Like contemporary and earlier samples from Iran/Turan

282 we find no evidence of Steppe-pastoralist-related ancestry in these samples. In contrast to all

283 other Iran/Turan samples, we find that these individuals also had negligible Anatolian

284 agriculturalist-related admixture, suggesting that they might be migrants from a population

285 further east along the cline of decreasing Anatolian agriculturalist ancestry. While we do not

286 have access to any DNA directly sampled from the Indus Valley Civilization (IVC), based on (a)

287 archaeological evidence of material culture exchange between the IVC and both BMAC to its

288 north and Shahr-i-Sokhta to its east (27), (b) the similarity of these outlier individuals to post-

289 IVC Swat Valley individuals described in the next section (27), (c) the presence of substantial

290 AASI admixture in these samples suggesting that they are migrants from South Asia, and (d) the

291 fact that these individuals fit as ancestral populations for present-day Indian groups in $q p A d m$

292 modeling, we hypothesize that these outliers were recent migrants from the IVC. Without ancient

293 DNA from individuals buried in IVC cultural contexts, we cannot rule out the possibility that the

294 group represented by these outlier individuals, which we call Indus_Periphery, was limited to the

295 northern fringe and not representative of the ancestry of the entire Indus Valley Civilization

296 population. In fact, it was certainly the case that the peoples of the Indus Valley were genetically

297 heterogeneous as we observe one of the Indus_Periphery individuals having $\sim 42 \%$ AASI

298 ancestry and the other two individuals having $\sim 14-18 \%$ AASI ancestry (but always mixes of the

299 same two proximal sources of $A A S I$ and Iranian agriculturalist-related ancestry). Nevertheless,

300 these results show that Indus_Periphery were part of an important ancestry cline in the wider

301 Indus region in the $3^{\text {rd }}$ millennium and early $2^{\text {nd }}$ millennium BCE. As we show in what follows,

302 peoples related to this group had a pivotal role in the formation of subsequent populations in

303 South Asia. 
305 Using a newly developed approach for estimating dates of admixture in ancient genomes (an adaptation of a previous method to measure ancestry covariance among pairs of neighboring positions in the genome; Supplementary Materials), we estimate that the time of admixture 308 between Iranian agriculturalist-related ancestry and AASI ancestry in the three Indus_Periphery 309 samples was $53 \pm 15$ generations ago on average, corresponding to a $95 \%$ confidence interval of 310 about $4700-3000 \mathrm{BCE}$ assuming 28 years per generation (28). This places a minimum date on the

311 first contact between these two types of ancestries.

\section{The Steppe}

314 Three individuals from the West Siberian forest zone with direct dates ranging from $6200 \mathrm{BCE}$ 315 to $4000 \mathrm{BCE}$ play an important role in this study as they are representatives of a never-before316 reported mixture of ancestry that we call West_Siberian_HG: 30\% derived from EHG, 50\%

317 from Ancestral North Eurasians (defined as being related deeply to 22000-15000 BCE Siberians $318(29,30))$, and $20 \%$ related to present-day East Asians. This ancestry type also existed in the 319 southern Steppe and in Turan, as it formed about $80 \%$ of the ancestry of an early $3^{\text {rd }}$ millennium

320 BCE agro-pastoralist from Dali, Kazakhstan, and also contributed to multiple outlier individuals 321 from $2^{\text {nd }}$ millennium sites in Kazakhstan and Turan (Fig. 2).

323 Using the West_Siberian_HG individuals as a reference population along with other pre324 Chalcolithic groups that have been previously reported in the ancient DNA literature, we 325 document the presence of a genetically relatively homogeneous population spread across a vast 326 region of the eastern European and trans-Ural Steppe between 2000-1400 BCE (Steppe_MLBA) 327 (17). Many of the samples from this group are individuals buried in association with artifacts of 328 the Corded Ware, Srubnaya, Petrovka, Sintashta and Andronovo complexes, all of which 329 harbored a mixture of Steppe_EMBA ancestry and ancestry from European Middle Neolithic agriculturalists (Europe_MN). This is consistent with previous findings showing that following

331 westward movement of eastern European populations and mixture with local European

332 agriculturalists, there was an eastward reflux back beyond the Urals $(6,16,31)$. Our new dataset 333 enhances our understanding of the Steppe_MLBA cluster by including many sites in present-day 334 Kazakhstan and as far east as the Minusinsk Basin of Russia—and in doing so allows us to 
335

appreciate previously undetected substructure. All previously reported samples fall into a subcluster we call Steppe_MLBA_West that harbors 26\% Europe_MN ancestry and $\sim 7 \%$ Steppe_EMBA ancestry. With our newly reported data we now also detect a previously unappreciated subcluster, Steppe_MLBA_East, which is significantly differentiated $\left(\mathrm{p}=7 \times 10^{-6}\right.$ from $q p A d m$ ), with $\sim 8 \%$ West_Siberian_HG-related ancestry and proportionally less of the other ancestry components, suggesting that people carrying Steppe_MLBA_West ancestry admixed with West_Siberian_HG-related peoples as they spread further east.

As in Iran/Turan, the outlier individuals provide key additional information.

First, our analysis of 50 newly reported individuals from the Kamennyi Ambar V cemetery from the Sintashta culture reveals three groups of outliers, in addition to the main cluster of 40 individuals. These outliers have elevated proportions of Steppe_EMBA,West_Siberian_HG or East Asian-related ancestry (and direct dates that are contemporaneous with the other individuals), thereby showing that this fortified site harbored people of diverse ancestries living side-by-side.

Second, samples from three sites from the southern and eastern end of the Steppe dated to 16001500 BCE (Dashti-kozy, Taldysay and Kyzlbulak) show evidence of significant admixture from Iranian agriculturalist-related populations, demonstrating northward gene flow from Turan into the Steppe at the same time as there was southward movement of Steppe_MLBA ancestry through Turan and into South Asia. These findings are consistent with evidence of a high degree of human mobility both to the north and south along the Inner Asian Mountain Corridor (32, 33).

Third, we observe samples from multiple sites dated to 1700-1500 BCE (Maitan, Kairan, Oy_Dzhaylau and Zevakinsikiy) that derive up to $\sim 25 \%$ of their ancestry from a source related to present-day East Asians and the remainder from Steppe_MLBA. A similar ancestry profile became widespread in the region by the Late Bronze Age, as documented by our time transect from Zevakinsikiy and samples from many sites dating to 1500-1000 BCE, and was ubiquitous by the Scytho-Sarmatian period in the Iron Age (34). This observation decreases the probability that populations in the $1^{\text {st }}$ millennium $\mathrm{BCE}$ and $1^{\text {st }}$ millennium $\mathrm{CE}$ - including Scythians, 
366 Kushans, and Huns, sometimes suggested as sources for the Steppe ancestry influences in South 367 Asia today (17) — contributed to the majority of South Asians, which have negligible East Asian ancestry in our analysis. It is possible that there were unsampled groups in Central Asia with negligible East Asian admixture that could have migrated later to South Asia. However, at least some (possibly all) of the Steppe pastoralist ancestry in South Asia owes its origins to southward pulses in the $2^{\text {nd }}$ millennium BCE, as indeed we prove directly through our observation of this ancestry in the Swat Iron Age individuals dating to 1000 BCE (discussed further below).

\section{South Asia}

375 Previous work has shown that the Indian Cline - a gradient of different proportions of West

376 Eurasian related ancestry in South Asia — can be well modeled as having arisen from a mixture

377 of two statistically reconstructed ancestral populations (the $A N I$ and the $A S I$ ), which mixed

378 mostly after $2000 \mathrm{BCE}(35,36)$. Ancient DNA analysis has furthermore revealed that the

379 populations along the Indian Cline actually descend more deeply in time from at least three ancestral populations (17), with ancestry from groups related to early Iranian agriculturalists, Steppe_EMBA, and Onge.

To shed light on the mixture events that transformed this minimum of three ancestral populations

384 into two (the $A N I$ and $A S I$ ), we used qpAdm to search for triples of source populations - the $A A S I$, all sampled ancient Iran/Turan-related groups, and all sampled ancient Steppe groupsthat could fit as sources for South Asians. As South Asian test populations we used an Indian

387 Cline group with high ANI ancestry (Punjabi.DG), one with high ASI ancestry (Mala.DG), early 388 Iron Age Swat Valley samples (Swat Protohistoric Grave Type - SPGT), and Early Historic Swat 389 Valley samples (Butkara_IA). Fig. 3A shows that the only models that fit all four test South 390 Asians groups are combinations that involve the AASI, Indus_Periphery and Steppe_MLBA (in 391 the analyses that follow, we therefore pooled the Steppe_MLBA). The evidence that the

392 Steppe_MLBA cluster is a plausible source for the Steppe ancestry in South Asia is also 393 supported by Y chromosome evidence, as haplogroup R1a which is of the Z93 subtype common 394 in South Asia today $(37,38)$ was of high frequency in Steppe_MLBA (68\%) (16), but rare in

395 Steppe_EMBA (absent in our data). 
397 To obtain a richer understanding of the ancestry of the entire Indian Cline, we took advantage of 398 previously published genome-wide data from 246 ethnographically diverse groups from South

399 Asia (11), from which we sub-selected 140 groups that fall on a clear gradient in PCA to

400 represent the Indian Cline (the other groups either fall off the cline due to additional African or

401 East Asian-related ancestry or had small sample size or heterogeneous ancestry). The per-group

$402 q p A d m$ estimates for the proportions of ancestry from these three sources are statistically noisy.

403 We therefore developed new methodology that allows us to jointly fit the data from all Indian

404 Cline groups within a hierarchical model. The analysis confirms that the great majority of all

405 groups on the Indian Cline can be jointly modeled as a mixture of two populations, and the

406 analysis also produces an estimate of the functional relationship between the ancestry

407 components. Setting Steppe_MLBA to its smallest possible proportion of zero to estimate the 408 minimum fraction of Indus_Periphery ancestry that could have existed in the ASI, we obtain $409 \sim 39 \%$. Setting AASI to its smallest possible proportion of zero to estimate the maximal fraction 410 of Indus_Periphery ancestry that could have existed in the $A N I$, we obtain $\sim 72 \%$. In fact, we find 411 four tribal groups from southern India (Palliyar, Ulladan, Malayan, and Adiyan) with close to

412 the maximum mathematically allowed proportion of Indus_Periphery-related ancestry, and we

413 find a population in northern Pakistan (Kalash) with close to the minimum. Thus, nearly

414 unmixed descendants of the $A S I$ and $A N I$ exist as isolated groups in South Asia today.

416 We built an admixture graph using qpGraph co-modeling Palliyar (as a representative of the $417 A S I$ ) and Juang (an Austroasiatic speaking group in India with low West Eurasian-relatedness), 418 and show that it fits when the ASI have $27 \%$ Iranian agriculturalist-related ancestry and the 419 Juang also harbor ancestry from an AASI population without Iranian admixture (Fig. 3). This 420 model is also notable in showing that early Iranian agriculturalists fit without $A A S I$ admixture, 421 and thus the patterns we observe are driven by gene flow into South Asia and not the reverse 422 (Fig. 3; Supplementary Materials). The fitted admixture graph also reveals that the deep 423 ancestry of the indigenous hunter-gather population of India represents an anciently divergent 424 branch of Asian human variation that split off around the same time that East Asian, Onge and 425 Australian aboriginal ancestors separated from each other. This finding is consistent with a 426 model in which essentially all the ancestry of present-day eastern and southern Asians (prior to 
427 West Eurasian-related admixture) derives from a single eastward spread, which gave rise in a

428 short span of time to the lineages leading to AASI, East Asians, Onge, and Australians (19).

430 Using admixture linkage disequilibrium, we estimate a date of $107 \pm 11$ generations ago for the 431 Iranian agriculturalist and AASI-related admixture in the Palliyar, corresponding to a 95\% 432 confidence interval of 1700-400 BCE assuming 28 years per generation (28). This date is 433 consistent with a previous estimate of $110 \pm 12$ generations ago for the Kalash (39). These 434 results suggest that the $A S I$ and $A N I$ were both largely unformed at the beginning of the $2^{\text {nd }}$ 435 millennium BCE, and imply that the ASI may have formed in the course of the spread of West 436 Asian domesticates into peninsular India beginning around 3000 BCE (where they were 437 combined with local domesticates to form the basis of the early agriculturalist economy of South 438 India (40)), or alternatively in association with eastward spread of material culture from the 439 Indus Valley after the IVC declined (41). Further evidence for a Bronze Age formation of the 440 ASI comes from our analysis of Austroasiatic-speaking groups in India such as Juang, who have 441 a higher ratio of $A A S I$-to-Iranian agriculturalist-related ancestry than the $A S I$ (Fig. 3,

442 Supplementary Materials). Austroasiatic speakers likely descend from populations that arrived 443 in South Asia in the $3^{\text {rd }}$ millennium BCE (based on hill cultivation systems associated with the 444 spread of Austroasiatic languages (20)), and our genetic results show that when Austroasiatic 445 speakers arrived they mixed with groups with elevated ratios of $A A S I$ - to Iranian-agriculturalist446 related ancestry than are found in the $A S I$, showing that the $A S I$ had not yet overspread 447 peninsular India.

449 Finally, we examined our Swat Valley time transect from 1200 BCE to 1 CE. While the earliest 450 group of samples $(S P G T)$ is genetically very similar to the Indus_Periphery samples from the 451 sites of Gonur and Shahr-i-Sokhta, they also differ significantly in harboring Steppe_MLBA 452 ancestry $(\sim 22 \%)$. This provides direct evidence for Steppe_MLBA ancestry being integrated into 453 South Asian groups in the $2^{\text {nd }}$ millennium BCE, and is also consistent with the evidence of 454 southward expansions of Steppe_MLBA groups through Turan at this time via outliers from the 455 main $B M A C$ cluster from 2000-1500 BCE. Later samples from the Swat time transect from the $4561^{\text {st }}$ millennium BCE had higher proportions of Steppe and AASI derived ancestry more similar to 
that found on the Indian Cline, showing that there was an increasing percolation of Steppe derived ancestry into the region and additional admixture with the ASI through time.

\section{Implications for Archaeology and Linguistics}

Our evidence that a population with both Iranian agriculturalist and South Asian hunter gatherer ancestry (Indus_Periphery) was established in the $3^{\text {rd }}$ millennium BCE—and that its Iranian agriculturalist-related and $A A S I$ ancestry sources mixed at an average time of around 4700-3000 $\mathrm{BCE}$ - shows that this type of Iranian agriculturalist-related ancestry must have reached the Indus Valley by the $4^{\text {th }}$ millennium BCE. However, it is very possible that Iranian agriculturalistrelated ancestry was widespread in South Asia even earlier, as wheat and barley agriculture as well as goat and sheep herding spread into South Asia after the $7^{\text {th }}$ millennium BCE, as attested at sites such as Mehrgarh in the hills surrounding the Indus Valley (20, 21), and these domesticates could have been carried by movements of people. Regardless of when these agricultural species arrived, the genetic data show that Indus_Periphery-related ancestry contributed in large proportions to both the $A N I$ and $A S I$, and that these two groups both formed in the $2^{\text {nd }}$ millennium BCE, overlapping the decline of the IVC and major changes in settlement

473 patterns in the northern part of the Indian subcontinent (41). A parsimonious hypothesis is that as 474 Steppe_MLBA groups moved south and mixed with Indus_Periphery-related groups at the end of 475 the IVC to form the ANI, other Indus_Periphery-related groups moved further south and east to 476 mix with $A A S I$ groups in peninsular India to form the $A S I$. This is consistent with suggestions that the spread of the IVC was responsible for dispersing Dravidian languages (42-44), although scenarios in which Dravidian languages derive from pre-Indus languages of peninsular India are

481 Our results also shed light on the question of the origins of the subset of Indo-European 482 languages spoken in India and Europe (45). It is striking that the great majority of Indo-European 483 speakers today living in both Europe and South Asia harbor large fractions of ancestry related to 484 Yamnaya Steppe pastoralists (corresponding genetically to the Steppe_EMBA cluster), 485 suggesting that "Late Proto-Indo-European" - the language ancestral to all modern Indo486 European languages - was the language of the Yamnaya (46). While ancient DNA studies have documented westward movements of peoples from the Steppe that plausibly spread this ancestry 
to Europe $(5,31)$, there has not been ancient DNA evidence of the chain of transmission to South Asia. Our documentation of a large-scale genetic pressure from Steppe_MLBA groups in the $2^{\text {nd }}$ millennium BCE provides a prime candidate, a finding that is consistent with archaeological evidence of connections between material culture in the Kazakh middle-to-late Bronze Age

492 Steppe and early Vedic culture in India (46).

Our analysis also provides an entirely new line of evidence for a linkage between Steppe ancestry and Indo-European culture. When we used $q p A d m$ to test if a mixture of ANI and ASI is a fit to the data for all 140 Indian Cline groups, we found 10 groups with poor fits and a significantly elevated ratio of Steppe_MLBA- to Indus_Periphery-related ancestry compared to the expectation for the model $(\mathrm{Z} \geq 3)$. We found the strongest two signals in Brahmin_Tiwari $\left(\mathrm{p}=2 \times 10^{-5}\right)$ and Brahmin_UP $\left(\mathrm{p}=4 \times 10^{-5}\right)$, and more generally there was a striking enrichment of a $Z \geq 3$ signals in groups of traditionally priestly status in northern India (57\% of groups with $Z \geq 3$ were Brahmins or Bhumihars even though these groups comprised only $11 \%$ of the 74 groups we analyzed in northern India). Although the enrichment for Steppe ancestry is not found in the southern Indian groups, the Steppe enrichment in the northern groups is striking as Brahmins and Bhumihars are among the traditional custodians of texts written in early Sanskrit. A possible explanation is that the influx of Steppe_MLBA ancestry into South Asia in the mid- $2^{\text {nd }}$ millennium BCE created a meta-population of groups with different proportions of Steppe ancestry, with ones having relatively more Steppe ancestry having a central role in spreading early Vedic culture. Due to strong endogamy in South Asia - which has kept some groups isolated from their neighbors for thousands of years (35) — some of this substructure within

510 Indian population still persists.

512 We finally highlight a remarkable parallel between the prehistory of two sub-continents of

513 Eurasia: South Asia and Europe. In both regions, West Asian agricultural technology spread 514 from an origin in the Near East in the $7^{\text {th }}$ and $6^{\text {th }}$ millennia BCE (Fig. 4). In South Asia this 515 occurred via the Iranian plateau, and in Europe via western Anatolia, with the technological 516 spreads mediated in both cases by movements of people. An admixed population was then 517 formed by the mixing of incoming agriculturalists and resident hunter-gatherers - in South Asia 518 eventually giving rise to the Indus_Periphery and ASI and in Europe the Middle Neolithic 
519 genetic cluster Europe_MN. In both Europe and South Asia, populations related to the Yamnaya 520 Steppe pastoralists arrived after this agriculturalist and hunter-gatherer admixture took place, 521 interacting with local populations to produce mixed groups, which then mixed further with 522 already resident agriculturalist populations to produce genetic groupings such as those found 523 associated with Corded Ware and central European Bell Beaker artifacts in much of Europe, and 524 the ANI genetic cluster in South Asia. These mixed groups then mixed further to produce the 525 major gradients of ancestry in both regions. Future studies of populations from South Asia and 526 the linguistically related Iranian world will extend and add nuance to the model presented here. 


\section{Figure Legends}

Fig. 1 Overview of ancient DNA data. (A) Number of newly reported samples passing our analysis thresholds and their date range is shown by site. (B) Locations, color-coded by analysis grouping. (C) Projections of ancient samples onto PCA axes computed using present-day Eurasians. (D) ADMIXTURE analysis, with components maximized in West_Siberian_HG, Anatolian agriculturalists, Iranian agriculturalists, indigenous South Asians and WHG in blue, orange, teal, red and green, respectively. (E) Y-chromosome haplogroups. N, Neolithic; C, Chalcolithic; BA, Bronze Age; IA, Iron Age; H, Historic; E/M/L, Early/Middle/Late; o, outlier.

Fig. 2 Modeling results. (A) Admixture events originating from 7 "Distal" populations leading to the formation of the modern Indian cloud shown geographically. Clines or 2-way mixtures of ancestry are shown in rectangles, and clouds (3-way mixtures) are shown in ellipses. (B) A schematic model of events originating from 7 "Distal" populations leading to the formation of the modern Indian cline, shown chronologically. (C) Admixture proportions as estimated using $q p A d m$ for populations reflected in A and B.

Fig. 3 The Genomic Origins of Indians. (A) We used $q p A d m$ to model four groups that are representative of major sources of South Asian ancestry over the last few thousand years (Punjabi.DG, Mala.DG, SPGT, and Butkara_IA) as mixtures of Onge, an Iran/Turan-related population, and a Steppe-related group, and report the minimum p-value (highlighting cases at $\mathrm{p}>0.01$ ). The only working models involve a combination of Indus_Periphery and a Steppe_MLBA group (note that the Steppe_MLBA_West group includes a subset Sintashta_MLBA and Srubnaya). (B) For all 140 Indian Cline groups, we give Maximum $A$ Posteriori fits for this model. Significant outliers $(|\mathrm{Z}|>2)$ are shown, and include a cluster of Brahmins (filled circles) and Bhumihars (filled squares) with excess Steppe pastoralist-related ancestry compared to others with similar West Eurasian ancestry proportion. (C) Admixture graph fit supports Iranian agriculturalist-related admixture into South Asia but no gives evidence of AASI-related admixture into ancient Iran; dotted lines show admixture events. 
557 Fig. 4 A Tale of Two Subcontinents. The prehistory of South Asia and Europe are parallel in

558 both being impacted by two successive spreads, the first from the Near East after 7000 BCE

559 bringing agriculturalists who mixed with local hunter-gatherers, and the second from the Steppe

560 after 3000 BCE bringing people who spoke Indo-European languages and who mixed with those

561 they encountered during their migratory movement. Mixtures of these mixed populations then

562 produced the rough clines of ancestry present in both South Asia and in Europe today (albeit

563 with more variable proportions of local hunter-gatherer-related ancestry in Europe than in India),

564 which are (imperfectly) correlated to geography. The plot shows in contour lines the time of the

565 expansion of Near Eastern agriculture. Human movements and mixtures, which also plausibly

566 contributed to the spread of languages, are shown with arrows. 


\section{Table 1 Summary of Key Findings}

\section{Iran/Turan}

1. There was a west-to-east gradient of ancestry across Eurasia in the Chalcolithic and Bronze Ages, with more Anatolian agriculturalist-related ancestry in the west and more West_Siberian_HG or AASI-related ancestry in the east, all superimposed on primary ancestry related to early Zagros agriculturalists. The establishment of the Anatolian ancestry gradient corresponds to the spread of crop-based agriculture across this region, raising the possibility that people of Anatolian ancestry spread this technology east just as they helped spread it west into Europe. However, Anatolian agriculturalist-related ancestry is absent in the Indus_Periphery samples, showing that if such people were instrumental in bringing crop farming eastward to Iran, diffusion of ideas brought it further east to South Asia.

2. The primary population of the BMAC was largely derived from preceding local Chalcolithic peoples and had little if any Steppe pastoralist ancestry of the type that is ubiquitous in South Asia today. Instead of being a source for South Asia, the $B M A C$ received admixture from South Asia.

3. Outlier analysis shows no evidence of Steppe pastoralist ancestry in groups surrounding BMAC sites prior to $2100 \mathrm{BCE}$, but suggests that between 2100-1700 BCE, the BMAC communities were surrounded by peoples carrying such ancestry.

4. We document a distinctive ancestry type- $58 \%-86 \%$ Iranian agriculturalist-related ancestry with little Anatolian agriculturalist-related admixture, and $14 \%-42 \%$ AASI ancestry - that was present at two sites known to be in close cultural contact with the Indus Valley Culture (IVC). Combined with similar ancestry about a millennium later in the post-IVC Swat Valley, this documents an Indus_Periphery population during the flourishing of the IVC, which we show formed by admixture 4700-3000 BCE.

\section{The Steppe}

1. In the Kazakh Steppe and Minusinsk Basin during the Middle to Late Bronze Age, ancestry typical of pastoralists in the western Steppe (Steppe_MLBA_West) admixed with ancestry related to earlier West_Siberian_HG-related groups to form a distinctive Steppe_MLBA_East cluster.

2. Outlier analysis shows that by $1600 \mathrm{BCE}$ in the Middle to Late Bronze Age of the Kazakh Steppe, there were numerous individuals with admixture from Turan, providing genetic evidence of northward movement into the Steppe in this period.

3. By 1500 BCE, there were numerous individuals in the Kazakh Steppe with East Asian-related admixture, the same type of ancestry that was widespread by the Scythian period (34). This ancestry is hardly present in the two primary ancestral populations of South Asia- $A N I$ and $A S I$ - suggesting that Steppe ancestry widespread in South Asia derived from earlier southward movements.

\section{South Asia}

1. After exploring a wide range of models of present-day and ancient South Asia, we identify a unique class of models that fits geographically and temporally South Asians: a mixture of AASI, Indus_Periphery, and Steppe_MLBA. We reject $B M A C$ as a primary source of ancestry in South Asians.

2. A population of which the Indus_Periphery samples were a part played a pivotal role in the formation of the two proximal sources of ancestry in South Asia, the $A N I$ and $A S I$. Both ends of the Indian Cline had major components of Indus_Periphery admixture: $\sim 39 \%$ for the $A S I$ and $\sim 72 \%$ for the $A N I$. Today there are groups in South Asia with very similar ancestry to the $A S I$ and $A N I$.

3. Much of the formation of both the $A S I$ and $A N I$ occurred in the $2^{\text {nd }}$ millennium BCE. Thus, the events that formed both the $A S I$ and $A N I$ overlapped the decline of the IVC.

4. The $A S I$ were not a clade with the earlier hunter-gatherer populations of South Asia $(A A S I)$, but harbored significant amounts of ancestry related to early Iranian agriculturalists, likely transmitted though the IVC. 
bioRxiv preprint doi: https://doi.org/10.1101/292581; this version posted March 31, 2018. The copyright holder for this preprint (which was not certified by peer review) is the author/funder, who has granted bioRxiv a license to display the preprint in perpetuity. It is made available under aCC-BY-NC-ND 4.0 International license.

\section{Fig. 1 Overview of ancient DNA data}
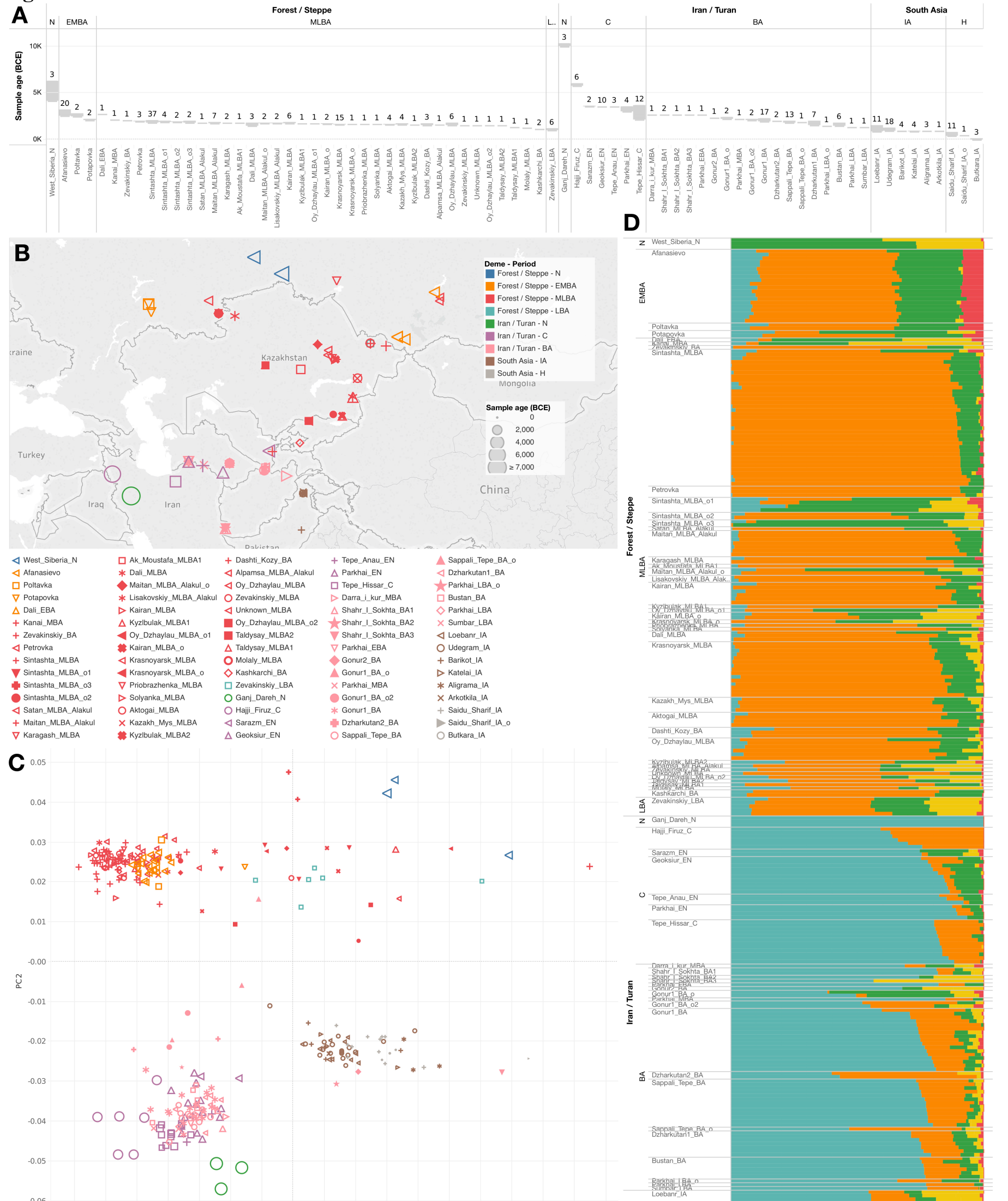
bioRxiv preprint doi: https://doi.org/10.1101/292581; this version posted March 31,2018. The copyright holder for this preprint (which was not certified by peer review) is the author/funder, who has granted bioRxiv a license to display the preprint in perpetuity. It is made available under aCC-BY-NC-ND 4.0 International license.

Fig. 2 Modeling results

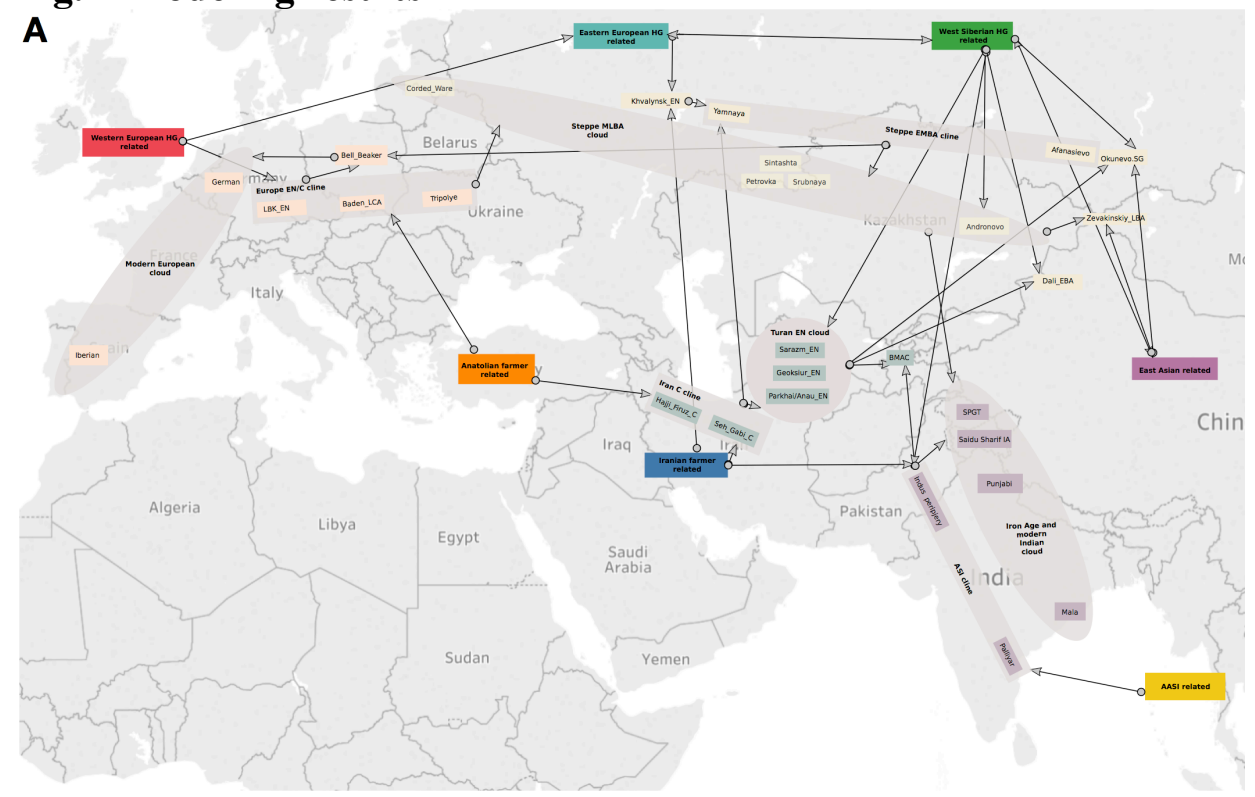

B

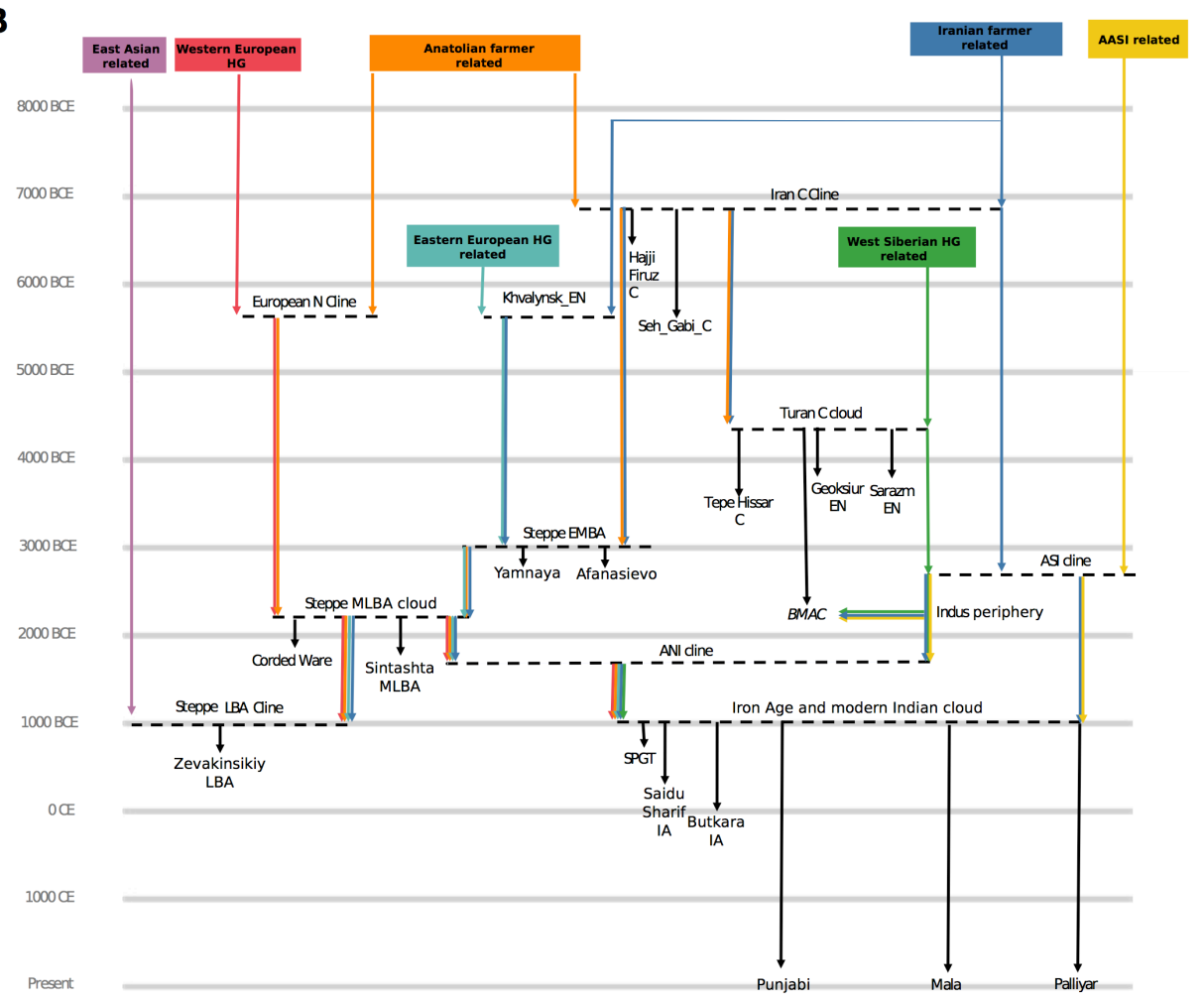

C

Deme - Period Label

Forest / Steppe - EN Khvalysnk_EN

Forest / Steppe - EMBA Steppe_EMBA

Dali_EBA

Forest / Steppe - MLBA Okunevo.SG

Steppe_MLBA_East

Steppe_MLBA_West

Forest / Steppe - LBA Zevakinsky_LBA

Iran / Turan - C

Haji_Firuz_C

Seh_Gabi_C

Tepe_Hissar_C

Parkhai/Anau_EN

Geoksiur_EN

Sarazm_EN

Iran / Turan - BA

BMAC

Shahr-i-Sokhta_BA1

Gonur2_BA

Shahr-i-Sokhta_BA2

South Asia - IA

Shahr-i-Sokhta_BA3 SPGT

South Asia - H

Saidu_Sharif_IA

Butkara_IA

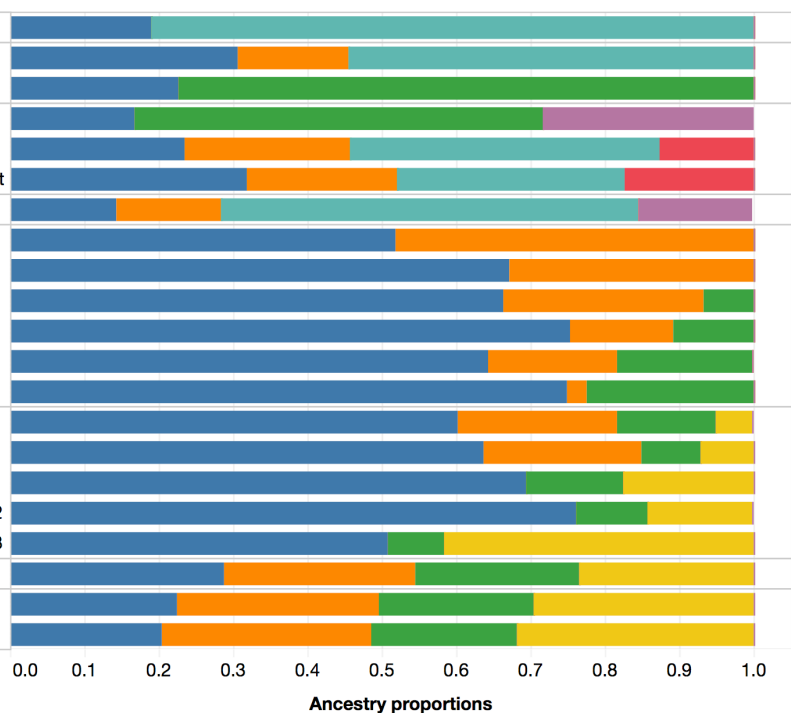


Fig. 3 The genomic origin of Indians

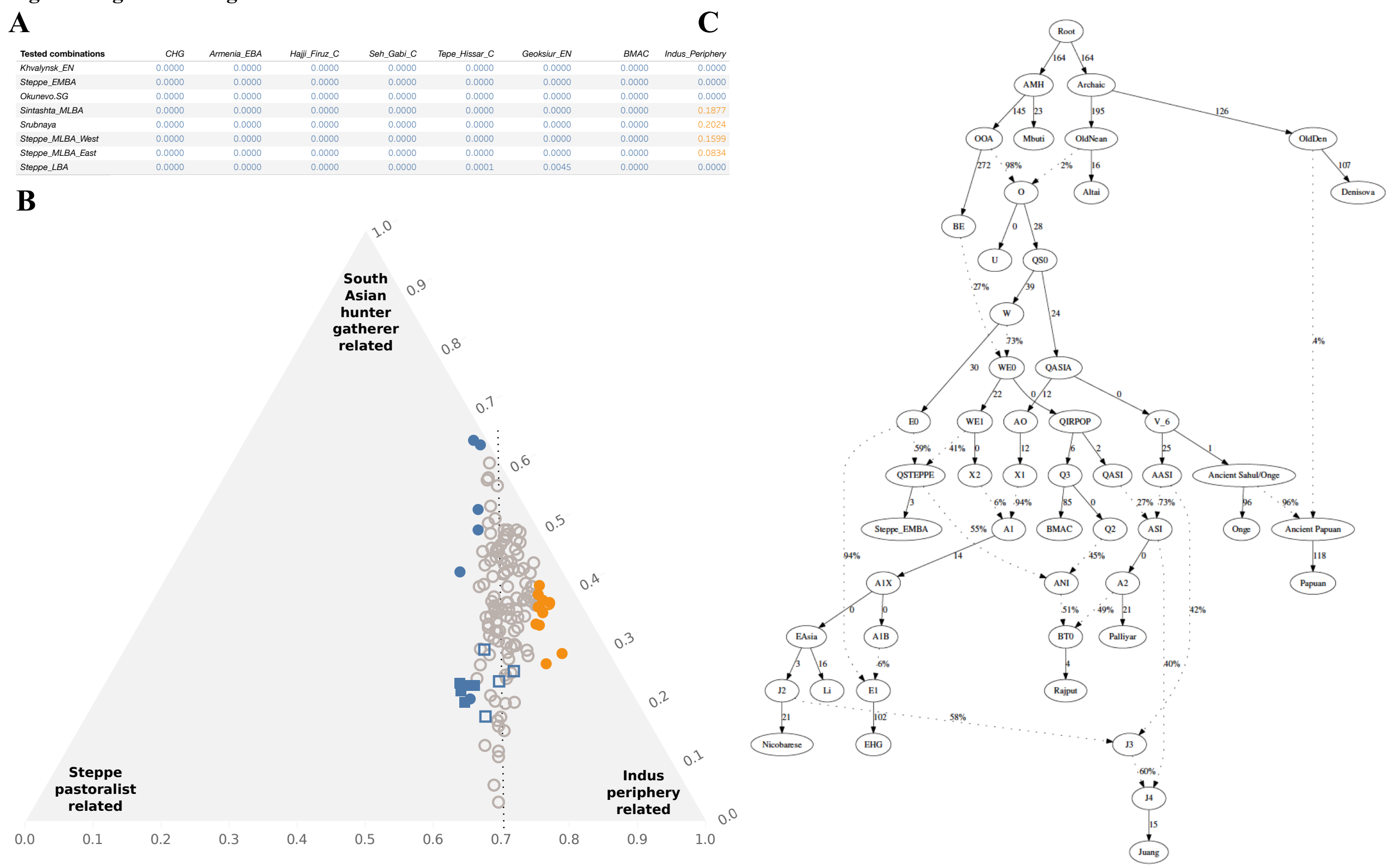


Fig. 4 A Tale of Two Subcontinents

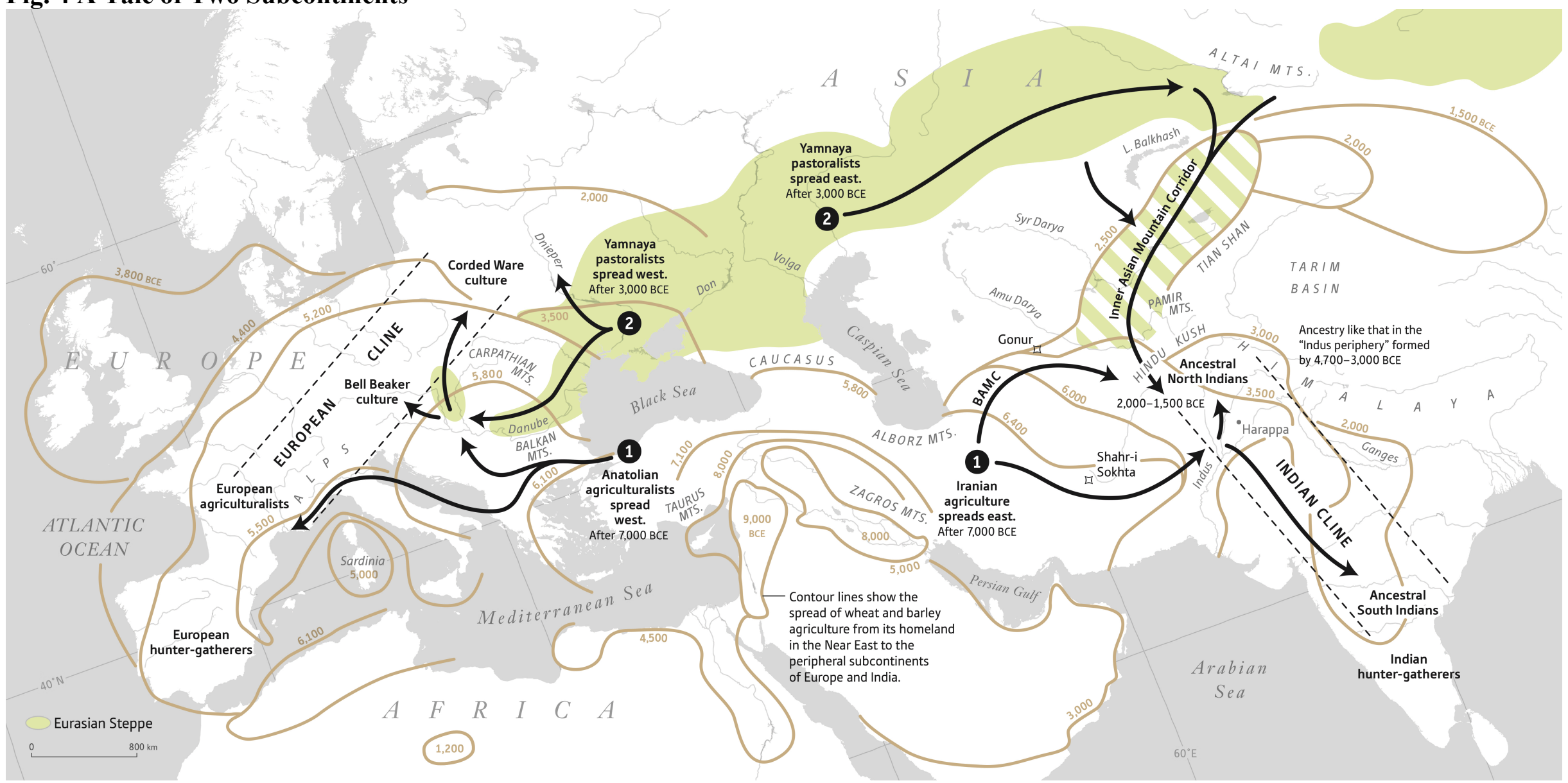


bioRxiv preprint doi: https://doi.org/10.1101/292581; this version posted March 31, 2018. The copyright holder for this preprint (which was not certified by peer review) is the author/funder, who has granted bioRxiv a license to display the preprint in perpetuity. It is made available under

Materials and Methods aCC-BY-NC-ND 4.0 International license.

Ancient DNA Laboratory Work We prepared powder from human skeletal remains either at field sites using a method for extracting petrous bone powder by drilling directly from the cranial base (47), or in dedicated clean rooms at Harvard Medical School, University College Dublin, or the Max Planck Institute for Evolutionary Anthropology in Leipzig Germany.

All the molecular work except for that of a single sample (Darra-i-Kur) was carried out at Harvard Medical School (HMS). At HMS, we extracted DNA using a method that is optimized to retain small DNA fragments $(1,2)$. We converted the DNA into a form that could be sequenced using a double-stranded library preparation protocol, usually pre-treating with the enzyme Uracil-DNA Glycosylase (UDG) to reduce the characteristic cytosine-to-thymine errors in ancient DNA (4). For some libraries, we substituted the MinElute columns used for cleaning up reactions with magnetic beads, and the MinElute column-based PCR cleanup at the end of library preparation with SPRI beads $(48,49)$. We enriched the libraries both for sequences overlapping mitochondrial DNA (50), and for sequences overlapping about 1.24 million nuclear targets after two rounds of enrichment (5, $6,8)$. We sequenced the enriched products on an Illumina NextSeq500 using v.2 150 cycle kits for $2 \times 76$ cycles and $2 \times 7$ cycles, and sequenced up to the point that the expected number of new SNPs covered per 100 additional read pairs sequenced was approximately less than 1 .

To analyze the data computationally, we separated read pairs into individuals based on searching for the expected two indices and two barcodes, allowing up to one mismatch from the expected sequence in each case. We removed adapters and merged together sequences requiring a 15 base pair overlap (allowing up to one mismatch), using a modified version of Seqprep (https://github.com/jstjohn/SeqPrep), which takes the highest quality base in the merged regions. We mapped the resulting single-ended sequences were mapped to the GRCh37 human reference (from the 1000 Genomes project) using the samse command of the Burrows-Wheeler Aligner tool $(B W A)$ (version 0.6.1) (51). We trimmed two nucleotides from the end of each sequence, and then randomly selected a single sequence at each site covered by at least one sequence in each individual to represent their genotype at that position ("pseudo-haploid" genotyping). For each sample we generated "pseudo-haploid" calls at the 1.24 million target sites, selecting sequences that have a minimum mapping quality of $\mathrm{MAPQ} \geq 10$, restricting to nucleotides with a minimum base quality of 20 , and trimming 2 base pairs from each end of the reads. 
bioRxiv preprint doi: https://doi.org/10.1101/292581; this version posted March 31, 2018. The copyright holder for this preprint (which was not certified by peer review) is the author/funder, who has granted bioRxiv a license to display the preprint in perpetuity. It is made available under

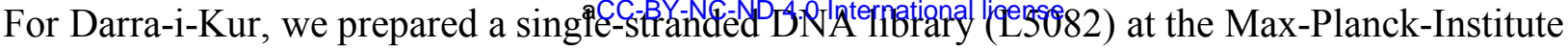

for Evolutionary Anthropology (MPI-EVA) in Leipzig, Germany, as part of a previous project (52).

The previous study only analyzed mitochondrial DNA, and for the current study, the library was enriched for molecules overlapping target the same panel of 1.24 million nuclear targets using two rounds of hybridization capture $(5,6,8)$. We sequenced the enriched libraries on 2 lanes of an Illumina HiSeq 2500 platform in a double index configuration ( $2 \times 76$ cycles) (53), and we called sites using Freelbis (54). We merged overlapping paired-end and trimmed using leeHom (55). We used $B W A$ to align the captured data to the human reference genome (GRCh37 from the 1000 Genomes project) (51). Only sequences showing a perfect match to the expected index combination evidence of contamination based on polymorphism on the $\mathrm{X}$ chromosome in males (57). were retained for downstream analyses.

We assessed evidence for ancient DNA authenticity by measuring the rate of damage in the first nucleotide (flagging individuals as potentially contaminated if they had a less than $3 \%$ cytosine to thymine substitution rate in the first nucleotide for a UDG-treated library and less than $10 \%$ substitution rate for a non-UDG-treated library). We used contamix to determine evidence of contamination based on polymorphism in mitochondrial DNA (56), and ANGSD to determine

Principal component analysis (PCA) We carried out PCA using the smartpca package of EIGENSOFT 7.2.1 (13). We used default parameters and added two options (lsqproject:YES and numoutlieriter:0 options) in order to project our ancient samples onto the PCA space. We used two basis sets for the projection: the first based on 1,340 present-day Eurasians genotyped on the Affymetrix Human Origins array, and the second based on a subset of 991 present-day West Eurasians $(5,10,58)$. These projections are shown repeatedly in the Supplementary Materials, and the whole-Eurasian projection is shown in Fig. 1. As part of this analysis, we also computed the $\mathrm{F}_{\mathrm{ST}}$ between groups using the parameters inbreed:YES and fstonly:YES.

ADMIXTURE clustering analysis Using PLINK2 (59), we first pruned our dataset using the -geno 0.7 option to ensure that we only performed our analysis on sites that had at least $70 \%$ of samples with a called genotype. We then ran ADMIXTURE (14) with 10 replicates, reporting the replicate with the highest likelihood. We show results for $\mathrm{K}=6$ in Fig. 1, as we found in practice that this provides the most resolution for disambiguating the sources of pre-Chalcolithic ancestry in our newly reported samples. 
bioRxiv preprint doi: https://doi.org/10.1101/292581; this version posted March 31, 2018. The copyright holder for this preprint (which was not certified by peer review) is the author/funder, who has granted bioRxiv a license to display the preprint in perpetuity. It is made available under

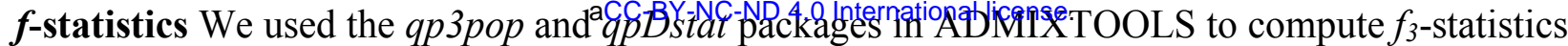

and $f_{4}$-statistics. We used the inbreed:YES parameter to compute $f_{3}$-statistics as a test for admixture

with an ancient population as a target, with all published and newly reported ancient genomes as

sources. Using the f4Mode:YES parameter in qpDstat, we also computed two sets of $f_{4}$-symmetry statistics to evaluate if pairs of populations are consistent with forming a clade relative to a comparison population. The first is a statistic where we compare all possible pairs of newly reported ancient groups (Reported1 and Reported2) to a panel of Test populations that encompass diverse pre-Chalcolithic and more widespread genetic variation (Test is one of Iran_Ganj_Dareh_Neolithic, Karelia_HG, Han, Onge, LBK_EN, AfontovaGora3, Ukraine_Mesolithic). Thus, we compute a statistic of the form $f_{4}$ (Reported1, Reported2; Test, Mbuti African outgroup). The second is a comparison of each newly reported group in turn against all possible pairs of Test populations, using statistics of the form $f_{4}($ Test 1, Test 2 ; Reported, Mbuti).

Formally modeling admixture history We used the $q p A d m$ methodology (5) in the

ADMIXTOOLS package to estimate the proportions of ancestry in a Test population deriving from a mixture of $N$ 'reference' populations by exploiting (but not explicitly modeling) shared genetic drift with a set of 'Outgroup' populations. We set the details:YES parameter, which reports a normally distributed Z-score for the fit (estimated with a block jackknife).

Hierarchical model of the Indian Cline We used $q p A d m$ as described above to obtain estimates for the proportion of Steppe-related, Iranian agriculturalist-related and $A A S I$-related ancestries and their relevant covariance matrices for each population on the Indian cline. We then jointly modeled these estimates using a bivariate normal model (since the three proportions sum to $100 \%$ ) and inferred the mean and covariance of the two parameters across all samples on the Indian cline using maximum likelihood estimation. Then, using this inferred matrix, we tested whether the cline could be modeled by a mixture of two populations, the $A N I$ and the $A S I$, in two ways. First, we examined whether the covariance matrix is singular, implying that knowledge of one estimated proportion of ancestry of one of the ancestry components revealed knowledge of the other two, as expected in a two-way mixture. Second, if we were able to establish that this was the case, we examined the difference between the expected and observed ratios of the ancestry proportions of individual populations in this generative model obtained from fitting all the populations simultaneously. This process resulted in a handful of populations deviating from expectation, as discussed in the main text and Supplementary Materials. 
bioRxiv preprint doi: https://doi.org/10.1101/292581; this version posted March 31, 2018. The copyright holder for this preprint (which was not certified by peer review) is the author/funder, who has granted bioRxiv a license to display the preprint in perpetuity. It is made available under

102 Supplementary Materials:

103 Materials and Methods

104 Online Tableau Server for visualizing data.

105 Data S1-S3

106 
bioRxiv preprint doi: https://doi.org/10.1101/292581; this version posted March 31, 2018. The copyright holder for this preprint (which was not certified by peer review) is the author/funder, who has granted bioRxiv a license to display the preprint in perpetuity. It is made available under

References and notes aCC-BY-NC-ND 4.0 International license.

108 1. J. Dabney et al., Complete mitochondrial genome sequence of a Middle Pleistocene cave bear reconstructed from ultrashort DNA fragments. Proceedings of the National Academy of Sciences of the United States of America 110, 15758-15763 (2013).

2. P. Korlevic et al., Reducing microbial and human contamination in DNA extractions from ancient bones and teeth. BioTechniques 59, 87-93 (2015).

3. M. Meyer et al., A high-coverage genome sequence from an archaic Denisovan individual. Science 338, 222-226 (2012).

4. N. Rohland, E. Harney, S. Mallick, S. Nordenfelt, D. Reich, Partial uracil-DNA-glycosylase treatment for screening of ancient DNA. Philosophical transactions of the Royal Society of London. Series B, Biological sciences 370, 20130624 (2015).

5. W. Haak et al., Massive migration from the steppe was a source for Indo-European languages in Europe. Nature 522, 207-211 (2015).

6. I. Mathieson et al., Genome-wide patterns of selection in 230 ancient Eurasians. Nature 528, 499-503 (2015).

7. I. Olalde et al., The Beaker phenomenon and the genomic transformation of northwest Europe. Nature 555, 190-196 (2018).

8. Q. Fu et al., An early modern human from Romania with a recent Neanderthal ancestor. Nature 524, 216-219 (2015).

9. N. J. Patterson et al., Ancient Admixture in Human History. Genetics 192, 1065-1093 (2012).

10. I. Lazaridis et al., Ancient human genomes suggest three ancestral populations for presentday Europeans. Nature 513, 409-413 (2014).

11. N. Nakatsuka et al., The promise of discovering population-specific disease-associated genes in South Asia. Nat. Genet. 49, 1403-1407 (2017).

12. N. Patterson, A. L. Price, D. Reich, Population structure and eigenanalysis. PLoS genetics 2, e190 (2006).

13. K. J. Galinsky et al., Fast Principal-Component Analysis Reveals Convergent Evolution of ADH1B in Europe and East Asia. American journal of human genetics 98, 456-472 (2016).

14. D. H. Alexander, J. Novembre, K. Lange, Fast model-based estimation of ancestry in unrelated individuals. Genome research 19, 1655-1664 (2009).

15. I. Olalde et al., Derived immune and ancestral pigmentation alleles in a 7,000-year-old Mesolithic European. Nature 507, 225-228 (2014).

16. I. Mathieson et al., The genomic history of southeastern Europe. Nature 555, 197-203 (2018).

17. I. Lazaridis et al., Genomic insights into the origin of farming in the ancient Near East. Nature 536, 419-424 (2016).

18. F. Broushaki et al., Early Neolithic genomes from the eastern Fertile Crescent. Science, (2016).

19. S. Mallick et al., The Simons Genome Diversity Project: 300 genomes from 142 diverse populations. Nature 538, 201-206 (2016).

20. D. Q. Fuller, in Examining the Farming/Language Dispersal Hypothesis. (McDonald Institute for Archaeological Research, 2003), pp. 191-213.

21. D. Q. Fuller, in The evolution and history of human populations in South Asia, M. D. Petraglia, B. Allchin, Eds. (Springer, Dordrecht, The Netherlands, 2007), pp. 393-443.

22. M. Gallego-Llorente et al., The genetics of an early Neolithic pastoralist from the Zagros, Iran. Sci Rep 6, 31326 (2016). 
bioRxiv preprint doi: https://doi.org/10.1101/292581; this version posted March 31, 2018. The copyright holder for this preprint (which was not certified by peer review) is the author/funder, who has granted bioRxiv a license to display the preprint in perpetuity. It is made available under

23. G. Barker, Goucher, C., in The Cainbridge thorretionflistorse. Volume 2: A World with Agriculture, 12,000 BCE-500 CE, G. Barker, Goucher, C., Ed. (Cambridge University Press, Cambridge, 2015), pp. 1-25.

24. A. J. Ammerman, L. L. Cavalli-Sforza, The neolithic transition and the genetics of populations in Europe. (Princeton University Press, Princeton, N.J., 1984), pp. xv, 176 p.

25. C. J. Stevens et al., Between China and South Asia: A Middle Asian corridor of crop dispersal and agricultural innovation in the Bronze Age. The Holocene 26, 1541-1555 (2016).

26. L. Sverchkov, Tokhary, drevnie indoevropeytsy v tsentral'noy Azii. 2012, Ed., (SMI-ASIA, Tashkent).

27. G. L. Possehl, The Middle Asian Interaction Sphere: Trade and contact in the 3rd millennium BC. Expedition 49, 40-42 ( 2004).

28. P. Moorjani et al., A genetic method for dating ancient genomes provides a direct estimate of human generation interval in the last 45,000 years. Proceedings of the National Academy of Sciences of the United States of America 113, 5652-5657 (2016).

29. M. Raghavan et al., Upper Palaeolithic Siberian genome reveals dual ancestry of Native Americans. Nature 505, 87-91 (2014).

30. Q. Fu et al., The genetic history of Ice Age Europe. Nature advance online publication, (2016).

31. M. E. Allentoft et al., Population genomics of Bronze Age Eurasia. Nature 522, 167-+ (2015).

32. M. D. Frachetti, Multiregional Emergence of Mobile Pastoralism and Nonuniform Institutional Complexity across Eurasia. Current Anthropology 53, 2-38 (2012).

33. M. D. Frachetti, C. E. Smith, C. M. Traub, T. Williams, Nomadic ecology shaped the highland geography of Asia's Silk Roads. Nature 543, 193-198 (2017).

34. M. Unterlander et al., Ancestry and demography and descendants of Iron Age nomads of the Eurasian Steppe. Nature communications 8, 14615 (2017).

35. D. Reich, K. Thangaraj, N. Patterson, A. L. Price, L. Singh, Reconstructing Indian population history. Nature 461, 489-494 (2009).

36. P. Moorjani et al., Genetic evidence for recent population mixture in India. American journal of human genetics 93, 422-438 (2013).

37. P. A. Underhill et al., The phylogenetic and geographic structure of Y-chromosome haplogroup R1a. European journal of human genetics : EJHG 23, 124-131 (2015).

38. M. Silva et al., A genetic chronology for the Indian Subcontinent points to heavily sexbiased dispersals. BMC evolutionary biology 17, 88 (2017).

39. G. Hellenthal et al., The Kalash Genetic Isolate? The Evidence for Recent Admixture. American journal of human genetics 98, 396-397 (2016).

40. C. A. Murphy, Fuller, D. Q., in A Companion to South Asia in the Past, G. R. G. R. Schug, Walimbe, S.R., Ed. (2016).

41. L. Giosan et al., Fluvial landscapes of the Harappan civilization. Proceedings of the National Academy of Sciences of the United States of America 109, E1688-1694 (2012).

42. I. Mahadevan, Dravidian Proof of the Indus Script via the Rig Veda: A Case Study. Bulletin of the Indus Research Centre 4, (2014).

43. A. Parpola, The Roots of Hinduism : The Early Aryans and the Indus Civilization. (Oxford University Press, New York, 2015), pp. xvi, 363 pages.

44. V. Kolipakam et al., A Bayesian phylogenetic study of the Dravidian language family. Royal Society Open Science 5, (2018).

45. C. Renfrew, Archaeology and language : the puzzle of Indo-European origins. (Cambridge University Press, New York, 1988), pp. xiv, 346 p., 348 p. of plates. 
bioRxiv preprint doi: https://doi.org/10.1101/292581; this version posted March 31, 2018. The copyright holder for this preprint (which was not certified by peer review) is the author/funder, who has granted bioRxiv a license to display the preprint in perpetuity. It is made available under

46. D. W. Anthony, The horse, the wheet, and languatignal ligense bronze-age riders from the Eurasian steppes shaped the modern world. (Princeton University Press, Princeton, NJ, 2007), pp. xii, 553 p.

47. K. A. Sirak et al., A minimally-invasive method for sampling human petrous bones from the cranial base for ancient DNA analysis. BioTechniques 62, 283-289 (2017).

48. M. M. DeAngelis, D. G. Wang, T. L. Hawkins, Solid-phase reversible immobilization for the isolation of PCR products. Nucleic acids research 23, 4742-4743 (1995).

49. N. Rohland, D. Reich, Cost-effective, high-throughput DNA sequencing libraries for multiplexed target capture. Genome research, (2012).

50. T. Maricic, M. Whitten, S. Paabo, Multiplexed DNA sequence capture of mitochondrial genomes using PCR products. PloS one 5, e14004 (2010).

51. H. Li, R. Durbin, Fast and accurate long-read alignment with Burrows-Wheeler transform. Bioinformatics 26, 589-595 (2010).

52. K. Douka et al., Direct radiocarbon dating and DNA analysis of the Darra-i-Kur (Afghanistan) human temporal bone. Journal of human evolution 107, 86-93 (2017).

53. M. Kircher, S. Sawyer, M. Meyer, Double indexing overcomes inaccuracies in multiplex sequencing on the Illumina platform. Nucleic acids research 40, e3 (2012).

54. G. Renaud, M. Kircher, U. Stenzel, J. Kelso, freeIbis: an efficient basecaller with calibrated quality scores for Illumina sequencers. Bioinformatics 29, 1208-1209 (2013).

55. G. Renaud, U. Stenzel, J. Kelso, leeHom: adaptor trimming and merging for Illumina sequencing reads. Nucleic acids research 42, e141 (2014).

56. Q. Fu et al., A revised timescale for human evolution based on ancient mitochondrial genomes. Current biology : $C B$ 23, 553-559 (2013).

57. T. S. Korneliussen, A. Albrechtsen, R. Nielsen, ANGSD: Analysis of Next Generation Sequencing Data. BMC bioinformatics 15, 356 (2014).

58. N. Patterson et al., Ancient admixture in human history. Genetics 192, 1065-1093 (2012).

59. C. C. Chang et al., Second-generation PLINK: rising to the challenge of larger and richer datasets. Gigascience 4, 7 (2015). 
bioRxiv preprint doi: https://doi.org/10.1101/292581; this version posted March 31, 2018. The copyright holder for this preprint (which was not certified by peer review) is the author/funder, who has granted bioRxiv a license to display the preprint in perpetuity. It is made available under

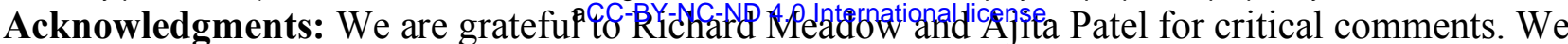
are grateful to the Minusinsk Regional Museum of N. M. Martyanov for sharing some of the skeletal samples analyzed in this study. We are grateful to Orazak Ismagulov and Ainagul Ismagulova for facilitating access to some of the Kazakh material. Funding: N.P. carried out this work while a fellow at the Radcliffe Institute for Advanced Study at Harvard University. P.M. was supported by a Burroughs Wellcome Fund CASI award. N.N. is supported by an NIGMS (GM007753) fellowship. T.C. and A.D. were supported by the Russian Science Foundation (project no. 14-50-00036). D.P., S.S. and D.L. were supported by European Research Council ERC-2011AdG 295733 grant (Langelin). M.R. acknowledges support from RFBR grant № 18-09-00779. Radiocarbon work supported by the NSF Archaeometry program BCS-1460369 to D.J.K. and B.J.C. and by the NFS Archaeology program BCS-1725067 to D.J.K. and T.Ha. K.T. was supported by the Council of Scientific and Industrial Research (CSIR), Government of India, New Delhi. N.B., A.N., and Z.M. were supported by the Max Planck Society. D.R. was supported by the U.S. National Science Foundation HOMINID grant BCS-1032255, the U.S. National Institutes of Health grant GM100233, by an Allen Discovery Center grant, and is an investigator of the Howard Hughes Medical Institute. Competing interests: The authors declare no competing interests. Data and materials availability: All sequencing data are available from the European Nucleotide Archive, accession number XXXXXXXX [to be made available on publication]. Genotype data obtained by random sampling of sequences at approximately 1.24 million analyzed positions or at approximately 600,000 positions (when merged with genotyping data from diverse present-day individuals) are available to researchers who write David Reich (reich@genetics.med.harvard.edu) a signed letter containing the following text: "For the data that is indicated as "signed letter only" (a) I will not distribute the data outside my collaboration; (b) I will not post the data publicly; (c) I will make no attempt to connect the genetic data to personal identifiers for the samples; (d) I will use the data only for studies of population history; (e) I will not use the data for any selection studies; (f) I will not use the data for medical or disease-related analyses; (g) I will not use the data for commercial purposes.", 